\title{
Analysis of fractional differential equations with fractional derivative of generalized Mittag-Leffler kernel
}

\author{
Mohammed Al-Refai', Abdalla Aljarrah¹ and Thabet Abdeljawad ${ }^{2,3,4^{*}}$ (D)
}

\section{"Correspondence:}

tabdeljawad@psu.edu.sa

${ }^{2}$ Department of Mathematics and

General Sciences, Prince Sultan

University, Riyadh, Saudi Arabia

${ }^{3}$ Department of Medical Research,

China Medical University, Taichung, 40402, Taiwan

Full list of author information is available at the end of the article

\begin{abstract}
In this paper, we study classes of linear and nonlinear multi-term fractional differential equations involving a fractional derivative with generalized Mittag-Leffler kernel. Estimates of fractional derivatives at extreme points are first obtained and then implemented to derive new comparison principles for related linear equations. These comparison principles are used to analyze the solutions of the linear multi-term equations, where norm estimates of solutions, uniqueness and several comparison results are established. For the nonlinear problem, we apply the Banach fixed point theorem to establish the existence of a unique solution.
\end{abstract}

MSC: 26A33; 34A08; 35B50; 33E12

Keywords: Fractional derivatives; Fractional differential equations; Maximum principle; Mittag-Leffler functions

\section{Introduction}

Several types of fractional derivatives have been introduced using different approaches. Recently, new types of fractional derivatives with nonsingular kernel have been developed and implemented in several applications; see [13, 14, 16, 29]. Abdeljawad and Baleanu [2] have developed a new type of fractional derivative with generalized Mittag-Leffler kernel that admits singular and nonsingular kernels based on some parameters. Fernandez et al. $[17,18]$ related the derivative to Prabhakar operators and expressed it in a series of Riemann-Liouville operators. The derivative has been implemented in mathematical modeling and there are few analytical and numerical studies $[1,17,18]$ devoted to this aspect. On the other hand, very recent work about the application of fixed point theory to integral equations, produced under the presence of different fractional operators, has been published $[4-6,12,20]$. This will be a motivation to a part of our recent work devoted to the nonlinear fractional case. For recent analysis techniques in ordinary, partial and fractional differential equations where Mittag-Leffler functions and their particular version the exponential functions we refer to [11, 15, 19, 21-24, 27]. For a generalized type of weighted fractional differences where the discrete Mittag-Leffler function plays an important role we refer to [3].

(c) The Author(s) 2021. This article is licensed under a Creative Commons Attribution 4.0 International License, which permits use sharing, adaptation, distribution and reproduction in any medium or format, as long as you give appropriate credit to the original author(s) and the source, provide a link to the Creative Commons licence, and indicate if changes were made. The images or other third party material in this article are included in the article's Creative Commons licence, unless indicated otherwise in a credit line to the material. If material is not included in the article's Creative Commons licence and your intended use is not permitted by statutory regulation or exceeds the permitted use, you will need to obtain permission directly from the copyright holder. To view a copy of this licence, visit http://creativecommons.org/licenses/by/4.0/. 
Definition 1.1 The left Caputo fractional derivative with generalized Mittag-Leffler kernel is defined by

$$
\left({ }^{A B C}{ }_{0} D^{\alpha, \beta} f\right)(t)=\frac{\mathbf{B}(\alpha)}{1-\alpha} \int_{0}^{t}(t-s)^{\beta-1} \mathbf{E}_{\alpha, \beta}\left[-\varepsilon_{\alpha}(t-s)^{\alpha}\right] f^{\prime}(s) d s,
$$

where $\mathbf{B}(\alpha)>0$ is a normalization function satisfying $\mathbf{B}(0)=\mathbf{B}(1)=1, \varepsilon_{\alpha}=\frac{\alpha}{1-\alpha}, 0<\alpha<1$, and $\mathbf{E}_{\alpha, \beta}$ is the Mittag-Leffler function of two parameters defined by

$$
\mathbf{E}_{\alpha, \beta}(x)=\sum_{k=0}^{\infty} \frac{x^{k}}{\Gamma(\alpha k+\beta)} .
$$

For $\beta \geq 1$, the kernel $k(t)=t^{\beta-1} \mathbf{E}_{\alpha, \beta}\left(-\varepsilon_{\alpha} t^{\alpha}\right)$ is nonsingular, and for $\beta=1$, we have the Atangana-Baleanu derivative. Here we are interested in the case of singular kernel, that is, $0<\beta<1$. For more details about the derivatives we refer the reader to [1, 2, 17, 18]. In this paper we consider the multi-term linear and nonlinear equations of the form

$$
\begin{aligned}
& a_{1} \frac{d v}{d t}+a_{2}{ }^{A B C}{ }_{0} D^{\alpha, \beta} v+q(t) v=h(t), \quad t>0, \\
& a_{1} \frac{d v}{d t}+a_{2}{ }^{A B C}{ }_{0} D^{\alpha, \beta} v=h(t, v), \quad t>0,
\end{aligned}
$$

where $0<\alpha, \beta<1, a_{1}, a_{2} \geq 0, a_{1}^{2}+a_{2}^{2}>0$. For $a_{2}=0$, and $a_{1}>0$, the above two equations reduce to first order linear and nonlinear differential equations and the theory of such equations is well-developed. So, we are interested here in $a_{2}>0$. Recently, several maximum-minimum principles were derived and implemented to study fractional differential equations $[7-10,25]$. In this paper we extend the maximum principles techniques to analyze the solutions of problems (1.2)-(1.3).

We organize this paper as follows: In Sect. 2, we derive new estimates of the fractional derivative of a function at its extreme points. In Sect. 3, we develop new comparison principles and analyze the solutions of the linear multi-term equation. In Sect. 4, we establish existence and uniqueness results to the nonlinear multi-term equation via the Banach fixed point theorem. We close with some conclusions in Sect. 5.

\section{Estimates of the fractional derivatives at the extreme points}

The following results concerning the Mittag-Leffler function are essential to proceed.

Lemma $2.1[26,28,30]$ The following hold true:

1. $\mathbf{E}_{\alpha, \alpha}(-x)=-\alpha \frac{d}{d x} \mathbf{E}_{\alpha}(-x), \alpha \geq 0$.

2. For $\beta>\alpha>0$, we have

$$
\mathbf{E}_{\alpha, \beta}(-x)=\frac{1}{\alpha \Gamma(\beta-\alpha)} \int_{0}^{1}\left(1-t^{\frac{1}{\alpha}}\right)^{\beta-\alpha-1} \mathbf{E}_{\alpha, \alpha}(-t x) d t .
$$

3. $\mathbf{E}_{\alpha, \beta}(-x), x \geq 0$ is completely monotone for $\alpha, \beta>0$, if and only if, $0<\alpha \leq 1$, and $\beta \geq \alpha$. That is

$$
(-1)^{n} \frac{d^{n}}{d x^{n}}\left(\mathbf{E}_{\alpha, \beta}(-x)\right) \geq 0, \quad x \geq 0 .
$$


Proposition 2.1 For $0<\alpha<\beta<1$, the kernel

$$
k(x)=x^{\beta-1} \mathbf{E}_{\alpha, \beta}\left(-\varepsilon_{\alpha} x^{\alpha}\right),
$$

is monotone non-increasing for $x>0$.

Proof We have $k(x)=\eta_{1}(x) \eta_{2}(x)$, where $\eta_{1}(x)=x^{\beta-1} \geq 0$, is monotone non-increasing, and $\eta_{2}(x)=\mathbf{E}_{\alpha, \beta}\left(-\varepsilon_{\alpha} x^{\alpha}\right)$. From Eq. (2.2) $\eta_{2}(x)$ is monotone non-increasing. Since $\mathbf{E}_{\alpha}(x)$ is completely monotone, then $\mathbf{E}_{\alpha, \alpha}(-x) \geq 0$, and from Eq. (2.1) we have $\eta_{2}(x)=\mathbf{E}_{\alpha, \beta}\left(-\varepsilon_{\alpha} x\right) \geq 0$. Now $\eta_{1}$ and $\eta_{2}$ are both nonnegative and monotone non-increasing, so their product is monotone non-increasing. Indeed, $k^{\prime}(x)=\underbrace{\eta_{2}^{\prime}(x) \eta_{1}(x)}_{\leq 0}+\underbrace{\eta_{2}(x) \eta_{1}^{\prime}(x)}_{\leq 0} \leq 0$.

Lemma 2.2 Assume $f \in H^{1}(0,1)$ is a function attaining its maximum at a point $t_{0} \in(0,1]$ and $0<\alpha<\beta<1$. Then

$$
\left({ }^{A B C}{ }_{0} D^{\alpha, \beta} f\right)\left(t_{0}\right) \geq \frac{\mathbf{B}(\alpha)}{1-\alpha} t_{0}^{\beta-1} \mathbf{E}_{\alpha, \beta}\left[-\varepsilon_{\alpha} t_{0}^{\alpha}\right]\left(f\left(t_{0}\right)-f(0)\right) \geq 0 .
$$

Proof We shall make use of the auxiliary function $g(t)=f\left(t_{0}\right)-f(t), t \in[0,1]$. Then it follows that $g(t) \geq 0$, on $[0,1], g\left(t_{0}\right)=0$ and $\left({ }^{A B C}{ }_{0} D^{\alpha, \beta} g\right)(t)=-\left({ }^{A B C}{ }_{0} D^{\alpha, \beta} f\right)(t)$. We have

$$
\left({ }^{A B C}{ }_{0} D^{\alpha, \beta} g\right)\left(t_{0}\right)=\frac{\mathbf{B}(\alpha)}{1-\alpha} \int_{0}^{t_{0}}\left(t_{0}-s\right)^{\beta-1} \mathbf{E}_{\alpha, \beta}\left[-\varepsilon_{\alpha}\left(t_{0}-s\right)^{\alpha}\right] g^{\prime}(s) d s .
$$

Let

$$
\begin{aligned}
k_{0}(s) & =\left(t_{0}-s\right)^{\beta-1} \mathbf{E}_{\alpha, \beta}\left[-\varepsilon_{\alpha}\left(t_{0}-s\right)^{\alpha}\right]=\left(t_{0}-s\right)^{\beta-1} \sum_{k=0}^{\infty} \frac{\left(-\varepsilon_{\alpha}\right)^{k}\left(t_{0}-s\right)^{\alpha k}}{\Gamma(\alpha k+\beta)} \\
& =\sum_{k=0}^{\infty} \frac{\left(-\varepsilon_{\alpha}\right)^{k}\left(t_{0}-s\right)^{\alpha k+\beta-1}}{\Gamma(\alpha k+\beta)},
\end{aligned}
$$

then

$$
\begin{aligned}
\frac{d k_{0}}{d s} & =-\sum_{k=0}^{\infty} \frac{\left(-\varepsilon_{\alpha}\right)^{k}}{\Gamma(\alpha k+\beta)}(\alpha k+\beta-1)\left(t_{0}-s\right)^{\alpha k+\beta-2} \\
& =-\left(t_{0}-s\right)^{\beta-2} \sum_{k=0}^{\infty} \frac{\left(-\varepsilon_{\alpha}\right)^{k}}{\Gamma(\alpha k+\beta-1)}\left(t_{0}-s\right)^{\alpha k} \\
& =-\left(t_{0}-s\right)^{\beta-2} \mathbf{E}_{\alpha, \beta-1}\left[-\varepsilon_{\alpha}\left(t_{0}-s\right)^{\alpha}\right]
\end{aligned}
$$

is well defined for $s<t_{0}$. Since $k(x)=x^{\beta-1} \mathbf{E}_{\alpha, \beta}\left(-\varepsilon_{\alpha} x^{\alpha}\right)$ is monotone non-increasing, and $x=t_{0}-s$, we have $\frac{d k_{0}}{d s} \geq 0$. By integration by parts with

$$
u=\left(t_{0}-s\right)^{\beta-1} \mathbf{E}_{\alpha, \beta}\left[-\varepsilon_{\alpha}\left(t_{0}-s\right)^{\alpha}\right] \text { and } d v=g^{\prime}(s) d s,
$$

we have

$$
\left({ }^{A B C}{ }_{0} D^{\alpha, \beta} g\right)\left(t_{0}\right)=\frac{\mathbf{B}(\alpha)}{1-\alpha}\left(\left.\left(t_{0}-s\right)^{\beta-1} \mathbf{E}_{\alpha, \beta}\left[-\varepsilon_{\alpha}\left(t_{0}-s\right)^{\alpha}\right] g(s)\right|_{0} ^{t_{0}}-\int_{0}^{t_{0}} \frac{d k_{0}}{d s} g(s) d s\right)
$$




$$
\begin{aligned}
& =\frac{\mathbf{B}(\alpha)}{1-\alpha}\left(-t_{0}^{\beta-1} \mathbf{E}_{\alpha, \beta}\left[-\varepsilon_{\alpha} t_{0}^{\alpha}\right] g(0)-\int_{0}^{t_{0}} \frac{d k_{0}}{d s} g(s) d s\right), \\
& \leq-\frac{\mathbf{B}(\alpha)}{1-\alpha} t_{0}^{\beta-1} \mathbf{E}_{\alpha, \beta}\left[-\varepsilon_{\alpha} t_{0}^{\alpha}\right] g(0) .
\end{aligned}
$$

Note that since $g\left(t_{0}\right)=0$, by L'Hospital's rule we have

$$
\lim _{s \rightarrow t_{0}}\left(t_{0}-s\right)^{\beta-1} g(s)=\lim _{s \rightarrow t_{0}}(1-\beta)^{-1} g^{\prime}(s)\left(t_{0}-s\right)^{\beta}=0, \quad 0<\beta<1 .
$$

Thus,

$$
\left({ }^{A B C}{ }_{0} D^{\alpha, \beta} g\right)\left(t_{0}\right)=-\left({ }^{A B C}{ }_{0} D^{\alpha, \beta} f\right)\left(t_{0}\right) \leq-\frac{\mathbf{B}(\alpha)}{1-\alpha} t_{0}^{\beta-1} \mathbf{E}_{\alpha, \beta}\left[-\varepsilon_{\alpha} t_{0}^{\alpha}\right] g(0),
$$

or

$$
\left({ }^{A B C}{ }_{0} D^{\alpha, \beta} f\right)\left(t_{0}\right) \geq \frac{\mathbf{B}(\alpha)}{1-\alpha} t_{0}^{\beta-1} \mathbf{E}_{\alpha, \beta}\left[-\varepsilon_{\alpha} t_{0}^{\alpha}\right]\left(f\left(t_{0}\right)-f(0)\right),
$$

which completes the proof.

If we process instead $-f$, then we have

Lemma 2.3 Assume $f \in H^{1}(0,1)$ is a function attaining its minimum at a point $t_{0} \in(0,1]$ and $0<\alpha<\beta<1$. Then

$$
\left({ }^{A B C}{ }_{0} D^{\alpha, \beta} f\right)\left(t_{0}\right) \leq \frac{\mathbf{B}(\alpha)}{1-\alpha} t_{0}^{\beta-1} \mathbf{E}_{\alpha, \beta}\left[-\varepsilon_{\alpha} t_{0}^{\alpha}\right]\left(f\left(t_{0}\right)-f(0)\right) \leq 0 .
$$

Lemma 2.4 Let a function $f \in H^{1}(0, T)$ attain its maximum at a point $t_{0} \in(0,1]$ and $0<$ $\alpha<\beta<1$. If $f(t)$ is not identically constant function on $\left[0, t_{0}\right]$, then

$$
\left({ }^{A B C}{ }_{0} D^{\alpha, \beta} f\right)\left(t_{0}\right)>\frac{\mathbf{B}(\alpha)}{1-\alpha} t_{0}^{\beta-1} \mathbf{E}_{\alpha, \beta}\left[-\varepsilon_{\alpha} t_{0}^{\alpha}\right]\left(f\left(t_{0}\right)-f(0)\right) \geq 0 .
$$

Proof Since $f(t)$ is not constant, $g(t)=f\left(t_{0}\right)-f(t) \geq 0$, and it is not identically zero on $\left[0, t_{0}\right]$. Thus

$$
\int_{0}^{t_{0}} \frac{d k_{0}}{d s} g(s) d s>0
$$

and the result follows from Eq. (2.4).

\section{Comparison principles}

In this section, we make use of the results derived in Sect. 2 to obtain new comparison principles for linear multi-term fractional equations including fractional derivatives with generalized Mittag-Leffler kernels. Then we use these principles to establish a uniqueness result and pre-norm estimate of solutions to related fractional initial value problems. 
Lemma 3.1 Assume a function $v \in H^{1}(0,1) \cap C[0,1]$ satisfies the fractional inequality

$$
P_{\alpha, \beta}(v)=a_{1} \frac{d v}{d t}+a_{2}\left({ }_{0}^{A B C} D^{\alpha, \beta} v\right)(t)+q(t) v(t) \leq 0, \quad t>0,0<\alpha<\beta<1,
$$

where $q(t)>0$ is continuous on $[0,1]$. If $v(0) \leq 0$, then $v(t) \leq 0, t \in[0,1]$.

Proof Assume the result is untrue, since $v$ is continuous on $[0,1], v$ attains an absolute maximum at $t_{0} \geq 0$ with $v\left(t_{0}\right)>0$. Since $v(0) \leq 0$, we have $t_{0} \neq 0$. If $v(t)$ is identically constant on $\left[0, t_{0}\right]$, then

$$
\frac{d v}{d t}\left(t_{0}\right)=\left({ }^{A B C}{ }_{0} D^{\alpha, \beta} v\right)\left(t_{0}\right)=0, \quad q\left(t_{0}\right)>0,
$$

and thus

$$
P_{\alpha, \beta}(v)\left(t_{0}\right)=q\left(t_{0}\right) v\left(t_{0}\right)>0,
$$

which contradicts (3.1).

If $v(t)$ is not identically constant on $\left[0, t_{0}\right]$, then, by virtue of the result in Lemma 2.4, we have

$$
\frac{d v}{d t}\left(t_{0}\right)=0,\left({ }_{0}^{A B C} D^{\alpha, \beta} v\right)\left(t_{0}\right)>0
$$

and thus

$$
P_{\alpha, \beta}(v)\left(t_{0}\right)=q\left(t_{0}\right) v\left(t_{0}\right)>0,
$$

which contradicts (3.1).

Corollary 3.1 Let $v_{1}, v_{2} \in H^{1}(0,1) \cap C[0,1]$ be possible solutions to the fractional initial value problems

$$
\begin{aligned}
& a_{1} \frac{d \nu_{1}}{d t}+a_{2}\left({ }^{A B C}{ }_{0} D^{\alpha, \beta} v_{1}\right)(t)+q(t) v_{1}(t)=h_{1}(t), \quad t>0,0<\alpha<\beta<1, \\
& a_{1} \frac{d \nu_{2}}{d t}+a_{2}\left({ }^{A B C}{ }_{0} D^{\alpha, \beta} v_{2}\right)(t)+q(t) v_{2}(t)=h_{2}(t), \quad t>0,0<\alpha<\beta<1, \\
& v_{1}(0)=r_{1}, \quad v_{2}(0)=r_{2},
\end{aligned}
$$

where $q(t)>0, h_{1}(t), h_{2}(t)$ are continuous on $[0,1]$. If $h_{1}(t) \leq h_{2}(t)$ and $r_{1} \leq r_{2}$, then

$$
v_{1}(t) \leq v_{2}(t), \quad t \in[0,1]
$$

Proof Let $z=v_{1}-v_{2}$,

$$
\begin{gathered}
P_{\alpha, \beta}(z)=a_{1} \frac{d z}{d t}+a_{2}\left({ }_{0}^{A B C} D^{\alpha, \beta} z\right)(t)+q(t) z(t)=h_{1}(t)-h_{2}(t) \leq 0, \\
t>0,0<\alpha<\beta<1
\end{gathered}
$$


and $z(0)=r_{1}-r_{2} \leq 0$. Applying the result in Lemma 3.1 we have $z(t) \leq 0$, which completes the proof.

Lemma 3.2 Assume $v \in H^{1}(0,1)$ is a possible solution to

$$
a_{1} \frac{d v}{d t}+a_{2}\left({ }_{0}^{A B C} D^{\alpha, \beta} v\right)(t)+q(t) v(t)=h(t), \quad t>0,0<\alpha<\beta<1,
$$

where $q(t)>0$ is continuous on $[0,1]$. Then

$$
\|v\|_{[0,1]}=\max _{t \in[0,1]}|v(t)| \leq M=\max _{t \in[0,1]}\left\{\left|\frac{h(t)}{q(t)}\right|,|v(0)|\right\},
$$

provided that the maximum $M$ exists.

Proof We have $M \geq\left|\frac{h(t)}{q(t)}\right|$, or $M q(t) \geq|h(t)|, t \in[0,1]$. Let $v_{1}=v-M$, then

$$
\begin{aligned}
P_{\alpha, \beta}\left(v_{1}\right) & =a_{1} \frac{d v_{1}}{d t}+a_{2}\left({ }_{0}^{A B C} D^{\alpha, \beta} v_{1}\right)(t)+q(t) v_{1}(t) \\
& =a_{1} \frac{d v}{d t}+a_{2}\left({ }_{0}^{A B C} D^{\alpha, \beta} v\right)(t)+q(t)(v-M) \\
& =h(t)-q(t) M \leq|h(t)|-q(t) M \leq 0 .
\end{aligned}
$$

Since $v_{1}(0)=v(0)-M \leq 0$, by virtue of Lemma 3.1 we have $v_{1}=v-M \leq 0$, or

$$
v \leq M
$$

Analogously, let $v_{2}=-M-v$, then

$$
\begin{aligned}
P_{\alpha, \beta}\left(v_{2}\right) & =a_{1} \frac{d v_{2}}{d t}+a_{2}\left({ }_{0}^{A B C} D^{\alpha, \beta} v_{2}\right)(t)+q(t) v_{2}(t) \\
& =-a_{1} \frac{d v}{d t}-a_{2}\left({ }_{0}^{A B C} D^{\alpha, \beta} v\right)(t)-q(t)(-M-v) \\
& =-h(t)-q(t) M \leq 0,
\end{aligned}
$$

which together with $v_{2}(0)=-M-v(0) \leq 0$, implies $v_{2}=-v-M \leq 0$, or

$$
v \geq-M
$$

If we use both of (3.3) and (3.4), then we have $|v(t)| \leq M, t \in[0,1]$ and hence the result.

Lemma 3.3 The multi-term fractional initial value problem

$$
\begin{aligned}
& a_{1} \frac{d v}{d t}+a_{2}\left({ }_{0}^{A B C} D^{\alpha, \beta} v\right)(t)+q(t) u(t)=h(t), \quad t>0,0<\alpha<\beta<1, \\
& v(0)=v_{0}
\end{aligned}
$$

where $q(t)>0$ is continuous on $[0,1]$, possesses at most one solution $v(t) \in H^{1}(0,1)$. 
Proof Let $v_{1}$ and $v_{2}$ be possible solutions to (3.5)-(3.6). Define $z(t)=v_{1}(t)-v_{2}(t)$, then

$$
a_{1} \frac{d z}{d t}+a_{2}\left({ }_{0}^{A B C} D^{\alpha, \beta} z\right)(t)+q(t) z(t)=0, \quad z(0)=0
$$

Applying the result in Lemma 3.2, we have

$$
\|z\|_{[0,1]} \leq M=0
$$

which implies $z(t)=0$, on $[0,1]$ and completes the proof.

\section{The nonlinear equation}

We consider the nonlinear multi-term initial value problem

$$
\begin{aligned}
& a_{1} \frac{d v}{d t}+a_{2}\left({ }_{0}^{A B C} D^{\alpha, \beta} v\right)(t)=h(t, v), \quad t>0, \\
& v(0)=v_{0} .
\end{aligned}
$$

We apply Banach fixed point theorem to show the existence of a unique solution to the problem (4.1)-(4.2). Since

$$
\left({ }_{0}^{A B C} D^{\alpha, \beta} v\right)(t)=\left({ }^{A B R}{ }_{0} D^{\alpha, \beta} v\right)(t)-\frac{\mathbf{B}(\alpha)}{1-\alpha} v_{0} \mathbf{E}_{\alpha, \beta}\left(-\varepsilon_{\alpha} t^{\alpha}\right)
$$

see [2], Eq. (4.1) is equivalent to

$$
a_{1} \frac{d v}{d t}+a_{2}\left({ }_{0}^{A B R} D^{\alpha, \beta} v\right)(t)=a_{2} \frac{\mathbf{B}(\alpha)}{1-\alpha} v_{0} \mathbf{E}_{\alpha, \beta}\left(-\varepsilon_{\alpha} t^{\alpha}\right)+h(t, v), \quad t>0,
$$

or

$$
\begin{aligned}
& a_{1} \frac{d v}{d t}+a_{2} \frac{\mathbf{B}(\alpha)}{1-\alpha} \frac{d}{d t} \int_{0}^{t}(t-s)^{\beta-1} \mathbf{E}_{\alpha, \beta}\left(-\varepsilon_{\alpha}(t-s)^{\alpha}\right) v(s) d s \\
& \quad=a_{2} \frac{\mathbf{B}(\alpha)}{1-\alpha} v_{0} \mathbf{E}_{\alpha, \beta}\left(-\varepsilon_{\alpha} t^{\alpha}\right)+h(t, v) .
\end{aligned}
$$

Applying the integral operator to the above equations yields

$$
\begin{aligned}
a_{1} v(t)= & a_{1} v_{0}+a_{2} \frac{\mathbf{B}(\alpha)}{1-\alpha} v_{0} \int_{0}^{t} \mathbf{E}_{\alpha, \beta}\left(-\varepsilon_{\alpha} s^{\alpha}\right) d s \\
& -a_{2} \frac{\mathbf{B}(\alpha)}{1-\alpha} \int_{0}^{t}(t-s)^{\beta-1} \mathbf{E}_{\alpha, \beta}\left(-\varepsilon_{\alpha}(t-s)^{\alpha}\right) v(s) d s+\int_{0}^{t} h(s, v) d s .
\end{aligned}
$$

Theorem 4.1 For $0<\alpha<\beta<1$, and $h:[0, T] \times \mathbb{R} \rightarrow \mathbb{R}$ be a continuous function that satisfy the Lipschitz condition

$$
|h(t, u)-h(t, v)| \leq K(u-v), K>0, \quad \text { for all } u, v \in C(\mathbb{R})
$$

If $a_{2} \frac{\mathbf{B}(\alpha)}{1-\alpha} \frac{1}{\Gamma(\beta+1)} T^{\beta}+K T<1$, then the fractional initial value problem (4.1)-(4.2) has a unique solution on $H^{1}(0, T)$. 
Proof On $H^{1}(0, T)$ define the norm

$$
\|f\|=\sup _{t \in[0, T]}|f(t)|
$$

and consider the linear operator $T: H^{1}(0, T) \rightarrow H^{1}(0, T)$ defined by

$$
\begin{aligned}
L\left(a_{1} v(t)\right)= & a_{1} v_{0}+a_{2} v_{0} \frac{\mathbf{B}(\alpha)}{1-\alpha} \int_{0}^{t} \mathbf{E}_{\alpha, \beta}\left(-\varepsilon_{\alpha} s^{\alpha}\right) d s \\
& -a_{2} \frac{\mathbf{B}(\alpha)}{1-\alpha} \int_{0}^{t}(t-s)^{\beta-1} \mathbf{E}_{\alpha, \beta}\left(-\varepsilon_{\alpha}(t-s)^{\alpha}\right) v(s) d s \\
& +\int_{0}^{t} h(s, v) d s .
\end{aligned}
$$

Let $v_{1}, v_{2} \in H^{1}(0, T), t \in(0, T)$ then

$$
\begin{aligned}
\left|L\left(a_{1} v_{1}\right)-L\left(a_{1} v_{2}\right)\right| \\
=\mid-a_{2} \frac{\mathbf{B}(\alpha)}{1-\alpha} \int_{0}^{t}(t-s)^{\beta-1} \mathbf{E}_{\alpha, \beta}\left(-\varepsilon_{\alpha}(t-s)^{\alpha}\right)\left(v_{1}(s)-v_{2}(s)\right) d s \\
\quad+\int_{0}^{t}\left(h\left(s, v_{1}\right)-g\left(s, v_{2}\right)\right) d s \mid \\
\leq a_{2} \frac{\mathbf{B}(\alpha)}{1-\alpha}\left\|v_{1}-v_{2}\right\|\left\|\mathbf{E}_{\alpha, \beta}\left(-\varepsilon_{\alpha}(t-s)^{\alpha}\right)\right\| \int_{0}^{t}(t-s)^{\beta-1} d s+K\left\|v_{1}-v_{2}\right\| \int_{0}^{t} d s \\
=\left(a_{2} \frac{\mathbf{B}(\alpha)}{1-\alpha}\left\|\mathbf{E}_{\alpha, \beta}\left(-\varepsilon_{\alpha}(t-s)^{\alpha}\right)\right\| \frac{t^{\beta}}{\beta}+K t\right)\left\|v_{1}-v_{2}\right\| .
\end{aligned}
$$

Since $\mathbf{E}_{\alpha, \beta}(-x)$ is decreasing for $x>0$, we have $\mathbf{E}_{\alpha, \beta}\left(-\varepsilon_{\alpha}(t-s)^{\alpha}\right) \leq \mathbf{E}_{\alpha, \beta}(0)=\frac{1}{\Gamma(\beta)}, 0 \leq s \leq$ $t \leq T$, and thus

$$
\begin{aligned}
\left|L\left(a_{1} v_{1}\right)-L\left(a_{1} v_{2}\right)\right| & \leq\left(a_{2} \frac{\mathbf{B}(\alpha)}{1-\alpha} \frac{1}{\Gamma(\beta)} \frac{T^{\beta}}{\beta}+K T\right)\left\|v_{1}-v_{2}\right\| \\
& =\left(a_{2} \frac{\mathbf{B}(\alpha)}{1-\alpha} \frac{1}{\Gamma(\beta+1)} T^{\beta}+K T\right)\left\|v_{1}-v_{2}\right\| .
\end{aligned}
$$

Since $a_{2} \frac{\mathbf{B}(\alpha)}{1-\alpha} \frac{1}{\Gamma(\beta+1)} T^{\beta}+K T<1$, then $L$ is a contraction and by the contraction fixed point principle on Banach spaces, $L$ has a unique fixed point.

\section{Concluding remarks}

We have considered linear and nonlinear multi-term fractional differential equations with fractional derivative of Caputo type involving the kernel $k(t)=t^{\beta-1} \mathbf{E}_{\alpha, \beta}(t), 0<\alpha, \beta<1$. We have established several comparison principles for related fractional linear equations and inequalities and used them to analyze the solutions of the multi-term linear fractional differential equations. These results are obtained under the condition $0<\alpha<\beta<1$, which quarantines the monotonicity property of the kernel $k(t), t>0$. Whether the results are extendable for arbitrary $0<\alpha, \beta<1$ is left for a future work. For the nonlinear equation we have established existence and uniqueness results via the Banach fixed point theorem. 


\section{Acknowledgements}

The author T. Abdeljawad would like to thank Prince Sultan University for the moral support to this work through the research group Nonlinear Analysis Methods in Applied Mathematics (NAMAM) group number RG-DES-2017-01-17 and TAS Lab.

\section{Funding}

Funding source is not available.

\section{Availability of data and materials}

This is not applicable in our paper.

\section{Competing interests}

The authors declare that they have no competing interests.

\section{Authors' contributions}

All authors contributed this manuscript and approved the final version.

\section{Author details}

${ }^{1}$ Department of Mathematics, Yarmouk University, Irbid, Jordan. ${ }^{2}$ Department of Mathematics and General Sciences, Prince Sultan University, Riyadh, Saudi Arabia. ${ }^{3}$ Department of Medical Research, China Medical University, Taichung, 40402, Taiwan. ${ }^{4}$ Department of Computer Science and Information Engineering, Asia University, Taichung, Taiwan.

\section{Publisher's Note}

Springer Nature remains neutral with regard to jurisdictional claims in published maps and institutional affiliations.

Received: 3 March 2021 Accepted: 23 June 2021 Published online: 10 July 2021

\section{References}

1. Abdeljawad, T.: Fractional operators with generalized Mittag-Leffler kernels and their iterated differintegrals. Chaos, Interdiscip. J. Nonlinear Sci. 29, 023102 (2019). https://doi.org/10.1063/1.5085726

2. Abdeljawad, T., Baleanu, D.: On fractional derivatives with generalized Mittag-Leffler function. Adv. Differ. Equ. 2018 468 (2018)

3. Abdeljawad, T., Jarad, F., Atangana, A., Mohammed, P.O.: On a new type of fractional difference operators on h-step isolated time scales. J. Fract. Calc. Nonlinear Syst. 1(1), 46-74 (2021). https://doi.org/10.48185/jfcns.v1i1.148

4. Adiguzel, R.S., Aksoy, U., Karapınar, E., Erhan, I.M.: On the solution of a boundary value problem associated with a fractional differential equation. Math. Methods Appl. Sci.. https://doi.org/10.1002/mma.665

5. Afshari, H., Kalantari, S., Karapinar, E.: Solution of fractional differential equations via coupled fixed point. Electron. J. Differ. Equ. 2015, Article ID 286 (2015)

6. Afshari, H., Karapınar, E.: A discussion on the existence of positive solutions of the boundary value problems via $\psi$-Hilfer fractional derivative on b-metric spaces. Adv. Differ. Equ. 2020, Article ID 616 (2020)

7. Al-Refai, M.: Fractional differential equations involving Caputo fractional derivative with Mittag-Leffler non-singular kernel: comparison principles and applications. Electron. J. Differ. Equ. 36, 1 (2018)

8. Al-Refai, M.: Maximum principles for nonlinear fractional differential equations in reliable space. Prog. Fract. Differ. Appl. 6(2), 95-99 (2020)

9. Al-Refai, M., Abdeljawad, T.: Analysis of the fractional diffusion equations with fractional derivative of non-singular kernel. Adv. Differ. Equ. 2017, Article ID 315 (2017). https://doi.org/10.1186/s13662-017-1356-2

10. Al-Refai, M., Hajji, M.A.: Analysis of a fractional eigenvalue problem involving Atangana-Baleanu fractional derivative: a maximum principle and applications. Chaos, Interdiscip. J. Nonlinear Sci. 29, 013135 (2019). https://doi.org/10.1063/1.5083202

11. Al-Refai, M., Luchko, Y.: Analysis of fractional diffusion equations of distributed order: maximum principles and its applications. Analysis (2015). https://doi.org/10.1515/anly-2015-5011

12. Alqahtani, B., Aydi, H., Karapınar, E., Rakocevic, V.: A solution for Volterra fractional integral equations by hybrid contractions. Mathematics 7, 694 (2019)

13. Atangana, A., Baleanu, D.: New fractional derivatives with non-local and non-singular kernel: theory and applications to heat transfer model. Therm. Sci. 20, 763-769 (2016)

14. Atangana, A., Koca, I.: Chaos in a simple nonlinear system with Atangana-Baleanu derivatives with fractional order. Chaos Solitons Fractals 89, 447-454 (2016)

15. Bedi, P., Kumar, A., Abdeljawad, T., Khan, A.: Study of Hilfer fractional evolution equations by the properties of controllability and stability. Alex. Eng. J. 60(4), 3741-3749 (2021). https://doi.org/10.1016/j.aej.2021.02.014

16. Caputo, M., Fabrizio, M.: A new definistion of fractional derivative without singular kernel. Prog. Fract. Differ. Appl. 1(2), 73-85 (2015)

17. Fernandez, A., Abdeljawad, T., Baleanu, D.: Relations between fractional models with three-parameter Mittag-Leffler kernels. Adv. Differ. Equ. 2020, 186 (2020). https://doi.org/10.1186/s13662-020-02638-5

18. Fernandez, A., Baleanu, D., Srivastava, H.M.: Series representations for models of fractional calculus involving generalised Mittag-Leffler functions. Commun. Nonlinear Sci. Numer. Simul. 67, 517-527 (2019)

19. Georgiev, S.G., Zennir, K.: New results on IBVP for class of nonlinear parabolic equations. Adv. Theory Nonlinear Anal. Appl. 2(4), 202-216 (2018)

20. Karapınar, E., Fulga, A., Rashid, M., Shahid, L., Aydi, H.: Large contractions on quasi-metric spaces with an application to nonlinear fractional differential-equations. Mathematics 7, 444 (2019)

21. Khan, A., Gomez-Aguilar, J.F., Khan, T.S., Khan, H.: Stability analysis and numerical solutions of fractional order HIV/AIDS model. Chaos Solitons Fractals 122, 119-128 (2019) 
22. Khan, H., Khan, A., Chen, W., Shah, K.: Stability analysis and a numerical scheme for fractional Klein-Gordon equations. Math. Methods Appl. Sci. 42(2), 723-732 (2019)

23. Khan, H., Khan, A., Jarad, F., Shah, A.: Existence and data dependence theorems for solutions of an ABC-fractional order impulsive system. Chaos Solitons Fractals 131, 109477 (2020)

24. Kim, I.S.: Semilinear problems involving nonlinear operators of monotone type. Res. Nonlinear Anal. 2, 25-35 (2019)

25. Luchko, Y.: Maximum principle for the generalized time-fractional diffusion equation. J. Math. Anal. Appl. 351, 218-223 (2009)

26. Miller, K.S., Samko, S.: A note on the complete monotonocity of the generalized Mittag-Leffler function. Real Anal. Exch. 23(2), 753-755 (1998)

27. Phuong, N.D., Luc, N., Long, L.D.: Modified quasi boundary value method for inverse source biparabolic. Adv. Theory Nonlinear Anal. Appl. 4, 132-142 (2020)

28. Pollard, H.: The complete monotonic character of the Mittag-Leffler function $E_{\alpha}(-x)$. Bull. Am. Math. Soc. 54, 1115-1116 (1948)

29. Saad, B., Alkahtani, T:: Chua's circuit model with Atangana-Baleanu derivative with fractional order. Chaos Solitons Fractals 89, 547-551 (2016)

30. Schneider, W.R.: Completely monotone generalized Mittag-Leffler functions. Expo. Math. 14, 3-16 (1996)

\section{Submit your manuscript to a SpringerOpen ${ }^{\circ}$} journal and benefit from:

- Convenient online submission

- Rigorous peer review

- Open access: articles freely available online

- High visibility within the field

- Retaining the copyright to your article

Submit your next manuscript at $\boldsymbol{\Delta}$ springeropen.com 